\title{
Erratum to: Modification of Weld Metal with Tungsten Carbide and Titanium Nitride Nanoparticles in Twin Submerged Arc Welding
}

N. P. Aleshin ${ }^{a}$, M. V. Grigor'ev ${ }^{a}$, N. V. Kobernik ${ }^{b, *}$, R. S. Mikheev ${ }^{b}$ A. S. Pankratov ${ }^{a}$, A. V. Samokhin ${ }^{c}$, N. V. Alekseev ${ }^{c}$, and M. A. Sinaisky ${ }^{c}$

a "Welding and Testing" Research and Training Center, Bauman State Technical University, Moscow, 105005 Russia

${ }^{b}$ Bauman State Technical University, Moscow, 105005 Russia

${ }^{c}$ Baikov Institute of Metallurgy and Materials Science, Russian Academy of Sciences, Moscow, 119334 Russia *e-mail: koberniknv@bmstu.ru

Submitted February 5, 2019; accepted February 5, 2019

DOI: $10.1134 / \mathrm{S} 0018143919090017$

Date of receipt of the article should read:

Received February 27, 2018

The original article can be found online at https://doi.org/10.1134/S0018143918050028. 